\title{
Broadcasting Mechanism In Vanets For Successful Transmission: A Review
}

\author{
Er. Ankita Dhiman, M.Tech Scholar \\ , Department of Computer Science and Engineering \\ ACET, Amritsar, I.K.G PTU, Punjab, India
Er. Amarpreet Singh, Associate Professor and HOD
Department of Computer Science and Engineering ACET, Amritsar, I.K.G PTU, Punjab, India

\begin{abstract}
Ad hoc network is a part of wireless networking. MANETs and VANETs fall under the category of ad hoc networks. The MANET is the mobile ad hoc networks in which the nodes are self-organizing. The VANETs are also a type of ad hoc networks, but it operates on vehicular nodes such as cars, etc. IN this the communication between vehicular nodes are done by using the concept of broadcasting or dissemination. The term safety message dissemination or broadcasting refers to the sending a notification to all of the nodes in the network simultaneously. But broadcasting also has some issues like it can lead to broadcast storm, redundancy of messages, delay in message delivery, network traffic, etc. This study provides a brief introduction to the concept of ad hoc networks, MANETs and VANETS. The various broadcasting types are also represented in this paper. An overview of the work that had been done under this field by various researchers is also described in this.
\end{abstract}

Keywords - Wireless Network, Ad Hoc Networks, MANETs, VANETs, Broadcasting

\section{INTRODUCTION}

Ad Hoc Networks are the wireless networks, which poses the property of self-organizing or did not follow any physical infra to settle down in the environment. Nodes or hubs in specially appointed systems (Ad Hoc Networks) act as both client and router. A few uses of specially appointed systems could incorporate mechanical and business applications including helpful versatile information exchange [1] such as military and protect operations. As of late, developing advances, for example, remote sensor systems (WSNs), wearable computing, pervasive processing, Internet of Things, have a great extent added to a further push toward application possibilities of specially appointed systems [2]. Ad hoc Networks present the characterized attributes of open connect, dynamic topology, and dispersed operation.

Ad hoc Networks are considered as totally self-ruling remote brief systems built up utilizing the gathered mobile devices, principally for military, crisis and emergency situations, where no framework is accessible [3]. It is a gathering of versatile hubs which don't require a physical infrastructure or the existence of the network for security and convenient purpose [4]. Likewise, wireless or remote connections are powerless to connection assaults going from inactive listening in to dynamic meddling [5]. Dissimilar to hardwired systems with physical guard at firewalls and entryways, assaults on systems can originate from all headings and may focus on any node. Independent nodes have deficient physical assurance and can be caught, traded off, and captured effortlessly [6]. Interruptions from a compromised hub are more hazardous and significantly harder to recognize [7]. Harm incorporates releasing confidential data, intrusive message and imitating nodes or hubs, in this way violates the essential security necessities. All these imply that each hub must be set up to experience with a foe either directly or indirectly [8]. 


\section{TYPES OF ADHOC NETWORKS}

MANETs: MANETs stands for Mobile Adhoc Networks. MANET has a self-organizing characteristic that all mobile nodes mutually connect by radio link, and the procedure is random topology [2]. The network topology can be changed quickly by time. MANET is becoming increasingly mainstream in emergency situations such as war and disaster [3] [4]. Each mobile node can act as a router as well as a host, because cooperation is easy and efficient messages can be sent over the radio link without the need for an expensive network infrastructure.

VANETs: Vehicular Adhoc Networks are collaboration of wireless technology with vehicles. It is specifically mean for creating a link between mobile vehicles and other roadside modules or units. It is a type of MANETs which set up the communication link among various vehicular nodes and its near located apparatus. The communication or data transmission among vehicular nodes in VANETs can be achieved by using IEEE 802.11, WiMAX, and Bluetooth etc. VANETs are specifically employed to maintain security and safety on roads and it also controls the traffic on the basis of observations of real time information. VANETs are easy and simple to employ and manage. VANETs has wider range of applications in various fields such as industrialization, safety of people on roads, Vehicular Adhoc Networks have potential to transmit or broadcast the message to its all vehicular nodes in order to generate a notification or warning to the users about safety issues etc.

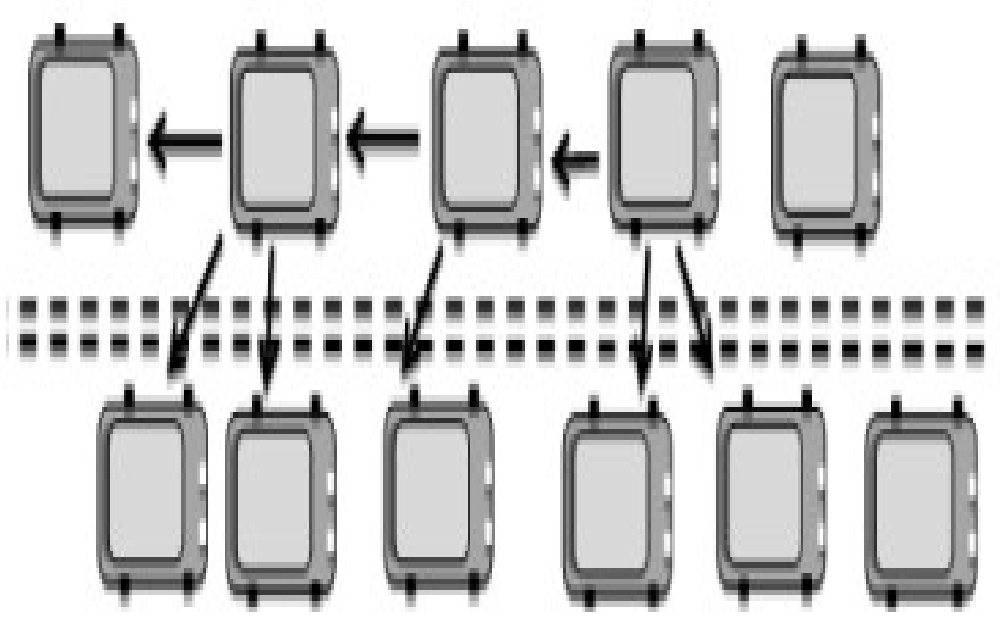

Figure 1. Vehicular Ad Hoc Networks [4]

\section{BROADCASTING}

Broadcasting is a term that refers to the process of transmitting the message to more than one node simultaneously. Broadcasting is a most appropriate process which distributes the safety message to all nodes in VANETs.

\subsection{Categorization of Broadcasting Mechanisms:}

The most common broadcasting mechanism that is used in VANETs is flooding. Flooding is a procedure of redelivering the safety notification to all the vehicular nodes in the network. But in this case, the issue of message redundancy and broadcasting storm can occur. These issues can be overtaken by using selective retransmission protocols which only let the selected vehicular nodes to broadcast the safety message.

The broadcasting protocols in VANETs can be divided into 6 types as depicted in following figure: 


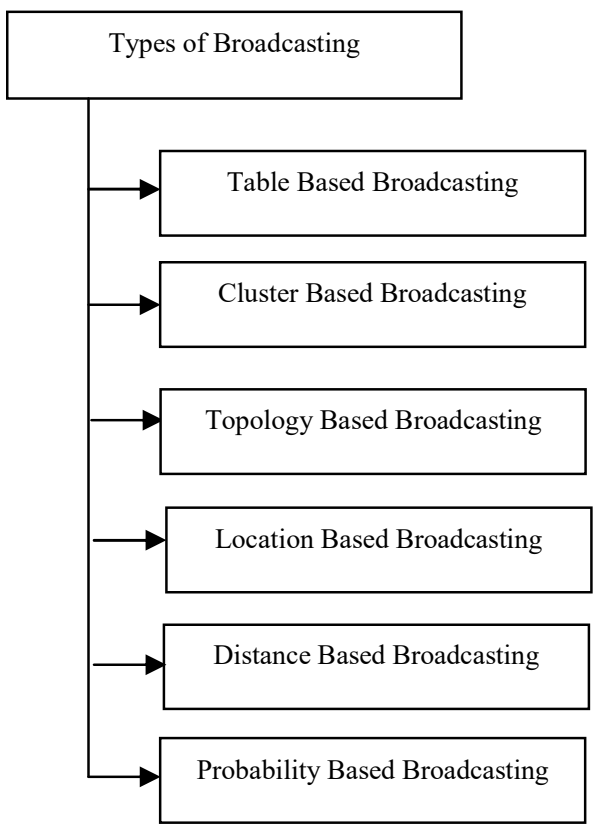

Figure 2: Types Of broadcasting

\subsubsection{Table Based Broadcasting:}

In table based broadcasting mechanism, it is mandatory for each and every node to maintain the information of adjacent node in a tabular format. This information table is updated corresponding to interval beacon message. The broadcasting mechanism that falls under the category of table based broadcasting method is as follows:

\subsubsection{Least Common Neighbor}

LCN is a table based message broadcasting protocol which works on the basis of selective flooding method which is utilized to promulgate emergency message in the network. In LCN the vehicular node which is going to broadcast the message in the network must append the list of its adjacent nodes in the message. After then the nodes which receive the broadcast message matches the listed neighbors with its own neighbors. The vehicle node which has at least common adjacent nodes as same in the message acted as a relay node.

\subsubsection{Track Detection}

Track detection is a protocol which is based on GPS broadcasting protocols. In this mechanism the vehicular nodes are categorized on the basis of different road groups such as same road ahead, various rods, and similar rods behind. Then form these road groups the farthest vehicular node is elected as a relay node.

\subsubsection{Cluster Based Broadcasting:}

In cluster based broadcasting, each and every vehicular node belongs to a cluster which is a group of related vehicular nodes. In this form of broadcasting, only the cluster head is permitted to broadcast the message .It is the responsibility of cluster heads to get assure about the delivery of the message to its cluster nodes. The various cluster head based broadcasting techniques are as follows:

\subsubsection{Topology Based Broadcasting:}

It is broadcasting process in which the topology of the network is followed in order to broadcast a message. The topology of network includes node connectivity, node density etc. The topology based broadcasting protocols are as follows: 
- $\quad$ DV-CAST (Distributed Vehicular broadcasting scheme)

- VDF (Vehicular Density Based Forwarding Broadcasting Scheme)

- $\quad$ STB (Spanning Tree Based Broadcasting Scheme)

\subsubsection{Location Based Broadcasting}

In location based broadcasting mechanism the safety message is disseminated only to a specific geographical location. In this the vehicular nodes assess the extra coverage region on the basis of their locations. The location is evaluated by using the concept of threshold. If the coverage is lesser than the threshold value that is pre-determined than the in such case node is not a candidate node to broadcast message. Following are the location based broadcasting protocols:

\subsubsection{UMB (Urban Multi-hop Broadcast)}

UMB stands for Urban Multi-Hop Broadcast protocol which is particularly meant to solve the various issue related to the urban VANETs i.e. redundant messages, reliability etc.

\subsubsection{AMB (Ad-Hoc Multi-hop Broadcasting)}

It is an extended version of UMB protocol which did not need to have any repeater as required in UMB.

\subsubsection{Distance based Broadcast}

In this type of broadcasting the broadcasting of safety notification is done on the basis of relative distance. In this a GPS device is employed on each and every node in order to evaluate the signal strength of adjacent node. Distance based broadcasting protocols are listed below:

\subsubsection{DDT(Distance Defer Transmission}

In this the node which is located at the farther distance from the sender node is elected as border node. The role of this border node is to sends the message to various nodes by following a shortest path to reach over there within a shortest delay time. Here the delay interval is inversely proportionate to the value corresponding to the distance of sender node.

\subsubsection{ODAM-C}

It is a distance based broadcasting protocol which is developed for increasing packet delivery rate by decreasing the packet loss ratio.

\subsubsection{O-CAST}

Its operating policies are same as the policies of DDT. Here in this the vehicular node with higher distance is elected as a relay node. It follows the same procedure as in DDT except the formulation for wait time evaluation.

\subsubsection{Probability based Broadcasting}

In this the relay node to re-disseminate the message is elected on the basis of predetermined value of probability function. This kind of broadcast is suitable for the network where the more than one node poses the same adjacent nodes. The protocols falls under this category is as below:

a. OAPB

b. REAR

\section{RELATED WORK}

OzanTonguz et al [1], "Broadcasting in VANETS", In this work the focus was lied on the concept of multi-hop broadcasting protocols specifically for VANETs. This study depicted that broadcasting in MANETs is different from broadcasting in VANETs. The reasons that were given behind this concept in this work was various parameters like structure of the network, mobility of employed nodes, traffic pattern with respect to various time interval etc. Hence it was proved that the traditional routing protocols are not suitable for VANETs. Three basic regimes were 
represented by the author which are mandatory for VANETs to work upon like high data traffic regime, sparse traffic regime and regular traffic regime. A specific routing protocol was designed for each and every regime. And DV-CAST was utilized to broadcast the message over the network.

Swati Chaturvedi et al [2], "A Study on Broadcasting in VANETs",this study represents that the evolution of technology has bring a drastic change in the way of communication and its means. VANETs are one of prominent area of wireless communication that is still moving towards enhancements. Its features such as self-organization of nodes ad movements of the nodes makes it more like to use by large number of users. Since the nodes in this network are vehicular nodes which always remain in a movement mode can't be stable. The VANETs are most likely to use in military field, for vehicle and road safety etc. by having a review to its features it is mandatory to follow a efficient broadcasting technique to achieve best of its operations. This study provides an overview to various broadcasting techniques that are followed in VANETs.

Shereen A.M. Ahmedet al [3], "A Survey on Broadcasting in VANETs", The objective of this study was to compare VANET broadcasting protocols. There are large number of broadcasting protocols that aims to provide high efficiency, reliability, scalability and reach ability. This study provides an overview to the various categorize of VANET broadcasting protocols on the basis of application for which those are used. The broadcasting categorize are further divided into various sub categorize on the basis of communication initiation, the criteria which is followed for electing the forwarding node. Quantitative evaluations were advised by performance metrics.

Sabih-urRehman et al [4], "Vehicular ad-Hoc networks (VANETs)—An overview and challenges",VANETs is a type of ad hoc network which is create by using vehicular nodes such as cars etc. In VANETs message dissemination method is followed to broadcast the message from sender node to other nodes in the network. There are lots of techniques and procedures which can be utilized to implement the broadcasting. Now, VANETs have become most prominent network which provides safety to the roadside networks. VANET has many benefits but also has some disadvantages also. This study represents an overview to the state of art of VANET and its issues. The concepts that were covered under this study was net6work structure, signal models etc. VANET satisfied all quality of service parameters. The concluded study showed that the ideal VANET is that which meets all the design parameters like QoS, lesser BER, high packet delivery ratio etc.

O.K. Tonguz ,et al [5], "DV-CAST: a distributed vehicular broad- cast protocol for vehicular ad hoc networks", This study proposed a source based VANET broadcasting mechanism which decreases the redundancy and node to node delay. In order to solve the issue of broadcast storm it is ideal that the node shoud be located at a farthest distance from the node which is elected to broadcast the message. In this study the neighbor table was used to find out the far away located node. The performance comparison was done with DV-CAST and the simulation was performed by implementing the SB-CAST protocol.

\section{IV.CONCLUSION AND FUTURE SCOPE}

The VANETs is a network that uses the vehicles as nodes in the network and mostly suitable in military field and for securing the vehicles from roadside accidents. Broadcasting is term that is used in VANETs which refers to deliver a message to more than one vehicle node at same time. But the broadcasting can also lead to various issues. Hence this study provides an overview to the broadcasting mechanism that is followed by VANETs.

For future scope a more prominent and advanced broadcasting mechanism can be utilized to achieve high performance message broadcasting and high reliability of the network. 


\section{REFERENCES}

[1] OzanTonguz et al, "Broadcasting in VANETS", IEEE, 2008

[2] Swati Chaturvedi et al, "A Study on Broadcasting in VANETs",IJSAR, Vol 5, Issue 6, PP 2273-2276, 2016ss

[3] Shereen A.M. Ahmedet al, "A Survey on Broadcasting in VANETs", Research Journal of Applied Sciences, Engineering and Technology, Vol 7, Issue 18, PP 3733-3739, 2014

[4] Sabih-urRehman et al, "Vehicular ad-Hoc networks (VANETs)—An overview and challenges", Computer Communications, vol. 48, pp. $141-158,2014$.

[5] O.K. Tonguz, N. Wisitpongphan, B. Fan , DV-CAST: a distributed vehicular broad- cast protocol for vehicular ad hoc networks, Wireless Commun. IEEE 17 (2) (2010) 47-57.

[6] M A. Amoroso , G. Marfia , M. Roccetti , Going realistic and optimal: a distributed multi-hop broadcast algorithm for vehicular safety, Comput. Netw. 55 (10) (2011) 2504-2519 .

[7] C. Wu , S. Ohzahata, T. Kato, A low latency path diversity mechanism for sender-oriented broadcast protocols in VANETs, Ad Hoc Networks 11 (7) (2013) 2059-2068

[8] Celimuge $\mathrm{Wu}$ et al, "Packet Size-Aware Broadcasting in VANETs With Fuzzy Logic and RL-Based Parameter Adaptation", IEEE, Vol 3, PP 2481-2491, 2015

[9] Celimuge $\mathrm{Wu}$ et al, "Efficient Broadcasting in VANETs Using Dynamic Backbone and Network Coding", IEEE, Vol 14, Issue 11, PP $6057-6071,2015$

[10] Razvan Cristian Voicu et al, "Fast and reliable broadcasting in VANETs using SNR with ACK decoupling", IEEE, 2014

[11] Amarpreet Singh ,Navneet Kaur "A Survey on Data Dissemination Protocols used in VANETs “ (International Journal of Computer Applications (0975 - 8887) Volume 120 - No.23, June 2015 Vehicular Adhoc Network (VANET)

[12] Rakesh Kumar et al, "A Review of various VANET data dissemination protocols", International Journal of u-and-e services and technology, Vol 5, Issue 3, Pp 1-18, 2012

[13] Arif Sari et al, "Review to the security issues in VANET", International journal on communication, network and system science, vol 8, Pp $552-566,2015$

[14] Shereen A.M. Ahmed et al, "Survey on broadcasting in VANETs", Research journal of Applied Science, engineering and technology, Vol 7, Issue 18, Pp 3733-3739, 2014

[15] Riyanks Chourse et al, "Review on vehicular Adhoc Network and broadcasting mechanism", International journal of scientific and engineering research, Vol 4, Issue 11, 2013

[16] Julio A. Sanguesa et al, "A Survey and Comparison study of broadcasting warning message dissemination schemes for VANETs", Hindawi, vol 2016, Pp 1-18, 2016

[17] Si Ho Cha et al, "A Survey of broadcasting protocols for VANETs", Smart computing Review, vol 4, Issue 4, Pp 1-10, 2014

[18] Sooksan Panichpapiboon et al, "A Review of information dissemination protocol for Vehicular Adhoc networks", IEEE, vol 14, Issue 3 , 2011

[19] A. Rahim et al, "A Comparative Study of Mobile and Vehicular Adhoc Networks", International Journal of recent trends in engineering, vol 2, Issue 4, Pp 1-3, 2009

[20] Xiaonin Ma et al, "Design and analysis of robust broadcast scheme for VANET safety related services", IEEE, vol 61, Issue 1, Pp 45-61, 2011

[21] Yu Tian Tsang et al, "A Vehicle density based forwarding scheme for emergency message broadcast in VANETs", IEEE, 2010

[22] Luciano Bononi et al, "A Cross Layered MAC and clustering scheme for efficient broadcast in VANETs", IEEE, 2008

[23] Peiyuan Lai et al, "A reliable broadcast routing scheme based on mobility prediction for VANETs", IEEE, 2009

[24] Sherali Zeadally et al, "Vehicular Ad hoc Network: status, results and challenges", Springer, vol 50, issue 4, Pp 217-241, 2012 